\title{
Antihypertensive drugs and new-onset diabetes in Thai Isan patients
}

\author{
Pornpun Chalermrum ${ }^{1}$, \\ Phisitt Vejakama², \\ Nonglek Kunawaradisai ${ }^{3}$, \\ Sawaeng Watcharathanakij ${ }^{3 *}$ \\ 1Department of Pharmacy, Phusing \\ Hospital, Sisaket, Thailand \\ ${ }^{2}$ Department of Internal Medicine, \\ Sunpasithiprasong Hospital, Ubon \\ Ratachathani, Thailand \\ ${ }^{3}$ Department of Pharmacy Practice, \\ Faculty of Pharmaceutical Science, Ubon \\ Ratchathani University, Ubon \\ Ratchathani, Thailand
}

*Corresponding author: Sawaeng Watcharathanakij sawaeng.w@ubu.ac.th

\section{KEYWORDS:}

Antihypertensive drugs; Newonset diabetes; Thai Isan patients

\begin{abstract}
Diabetes is a major global public health problem worldwide caused by many factors including genetics and medication. Antihypertensive drugs had been reported to be associated with new-onset diabetes (NOD) in many countries, but not in Thai patients with hypertension. The purpose of this study was to investigate the association between antihypertensive drugs and NOD among Thai Isan patients. This retrospective cohort study obtained data from electronic medical records from community hospitals in Ubon Ratchathani from January 1, 2007 through December 31, 2015. Newly diagnosed hypertensive patients aged $\geq$ 20 years who received ACEI-based, thiazide-based and combined regimen were identified by ICD 10 code (I10 - I15) and were followed up for 6 years or diagnosed with NOD (E10 - E14). Propensity score was used as a covariate to control for confounding by indication bias due to non-randomly selection process during routine clinical treatment of hypertensive patients. Kaplan-Meier analysis and multivariate cox regression with propensity score adjustment were used to investigate the incidence and the risk of NOD. Of 3,689 newly diagnosed hypertension patients enrolled, $396(10.73 \%)$ patients developed NOD during follow-up period. NOD incidence were 11.06 (95\%CI, 9.19 - 13.27), 14.17 (95\%CI, $12.48-16.05)$, and 14.17 (95\%CI, $11.53-17.35)$ in ACEI-based, thiazide-based and combined regimen users respectively. After adjusting for confounding by indication, the Hazard Ratios were $1.540(95 \% \mathrm{CI}, 0.963-2.463)$ for thiazide-based regimen and 1.341 (95\% CI, 0.769-2.338) for combined regimen compared to ACEIbased regimen. In conclusion, thiazide-based and combined regimen were not associated with NOD compared with ACEI-based regimen in Thai Isan patients.
\end{abstract}

\section{INTRODUCTION}

Diabetes is a major global public health problem that affected 425 million people, accounting for USD 727 billion dollars in health expenditure ( $12 \%$ of global total spending on adults $)^{1}$. It is also the risk factor of cardiovascular disease ${ }^{2}$ which is a leading cause of death in Thailand ${ }^{3,4}$. There are many risk factors for diabetes such as family history of diabetes, gestational diabetes history, physical activity and poly ovarian syndrome ${ }^{5-8}$. In addition both observational studies and network meta-analysis of clinical trials revealed that antihypertensive drugs were associated with new-onset of diabetes (NOD) $)^{9-12}$. Among antihypertensive drugs, 
diuretics were associated with a significant increase in the risk of $\mathrm{NOD}^{9-11}$ whereas angiotensin converting enzyme inhibitors (ACEI) were associated with a significant lower risk of NOD compared with diureics ${ }^{12}$. However, the evidence in Thai patients with hypertension has not been studied. The purpose of this study is to investigate the association between antihypertensive drugs regimens (ACEI based regimen, thiazide-based regimen and combined regimen) and NOD among Thai patients with hypertension.

\section{MATERIALS AND METHODS}

\subsection{Study design}

We performed a population based retrospective cohort study among patients aged $\geq$ 20 years old with newly diagnosed hypertension and had medication history $\geq 180$ days before the enrollment date. Newly diagnosed hypertension was identified by International classification of disease and related health problem $10^{\text {th }}$ revision (ICD10), I10 - I15 codes with prescribed antihypertensive drugs $\geq 2$ visits. Data were obtained from electronic medical records (EMRs) from 10 community hospitals, Ubon Ratchathani province from January 1, 2007 through December 31, 2015.

We excluded those who met the following criteria: 1) patients who were prescribed any antihypertensive drugs for other indications or antidiabetic drugs before diagnosed with hypertension, 2) patients who were diagnosed with diabetes before diagnosed with hypertension and 3) non Thai.

Due to hypertension treatment guideline and multiple drug class treatment in clinical setting, we identified three exposure groups: 1) ACEI-based regimen users, patients who were prescribed ACEI drug class alone or with any other antihypertensive drug class except thiazide diuretics drug class, 2) thiazide-based regimen users, patients who were prescribed thiazide diuretics drug class alone or with any other antihypertensive drug class except ACEI drug class and 3) combined regimen users, patients who were prescribed ACEI drug class and thiazide diuretics drug class without or with any other antihypertensive drug class.

The primary endpoint was NOD, identified by ICD10 (E10 - E14) with prescribed antidiabetic drugs. The primary endpoint of the study was defined as the NOD after 28 days of antihypertensive drugs treatment during followup period. We assumed that NOD within the 28 days after antihypertensive drugs use could not be attributable to treatment. From the entire EMRs, we enrolled those who were newly diagnosed hypertension from January 1, 2007 through December 31, 2009 into the study and followed up them for 6 years or until reached the endpoint.

\subsection{Statistical analysis}

Data were analyzed by descriptive analysis; mean for normally distributed variables, median (with $25-75 \%$ range) for non-normally distributed variables. Propensity scores were calculated by logistic regression to predict the probability of receiving treatment with antihypertensive drugs to adjust for confounding by indication. Univariate survival analysis for NOD status was performed using Kaplan Meier analysis. The effect of antihypertensive drugs on development of NOD was computed by time dependent cox regression in bivariate and multivariate analysis. Variables in multivariate model were age, sex, body mass index, blood pressure, total cholesterol, high density lipoprotein (HDL) cholesterol, low density lipoprotein (LDL) cholesterol, triglyceride, statin use and propensity score. We analyzed the multivariate data with 2 models; 1) multivariate analysis without propensity score and 2) multivariate analysis with propensity score as covariate adjustment. A $\mathrm{p}$-value $<0.05$ was considered statistically significant. All analyses were performed using STATA version 14.

\section{RESULTS}

\subsection{Subjects characteristics}

Of 13,664 newly diagnosed hypertensive patients identified during the enrollment period, 3,689 patients met the inclusion criteria and didn' $t$ meet the exclusion criteria. Of these eligible subjects, $43.43 \%$ were male. The average age of ACEI-based regimen users, thiazide-based regimen users and combined-regimen users when they were first diagnosed with hypertension were $58.29,57.13$ and 58.41 years respectively. There were no significant difference in age, body mass 
Table 1. Study population characteristics.

\begin{tabular}{|c|c|c|c|c|}
\hline Variables & $\begin{array}{l}\text { ACEI-based } \\
\text { regimen }^{\mathrm{a}}\end{array}$ & $\begin{array}{l}\text { Thiazide-based } \\
\text { regimen }^{\text {b }}\end{array}$ & Combined regimen $^{\mathrm{c}}$ & $p$-value \\
\hline Sex, male $(\%)$ & 46.53 & 41.81 & 43.11 & \\
\hline $\operatorname{Age}(y)^{*}$ & $58.29(13.54)$ & $57.13(13.32)$ & $58.41(13.51)$ & 0.359 \\
\hline $\operatorname{BMI}\left(\mathrm{kg} / \mathrm{m}^{2}\right)^{*}$ & $24.01(4.15)$ & $24.25(4.12)$ & $24.22(4.44)$ & 0.352 \\
\hline Systolic BP $(\mathrm{mmHg})^{*}$ & $158.91(20.89)$ & $158.51(16.95)$ & $168.68(19.83)$ & 0.008 \\
\hline Diastolic BP (mmHg) ${ }^{*}$ & $89.22(15.13)$ & $90.33(12.67)$ & $94.18(15.96)$ & 0.008 \\
\hline Fasting glucose $(\mathrm{mg} / \mathrm{dL})^{*}$ & $108.65(27.37)$ & $100.51(19.24)$ & $107.18(31.54)$ & 0.044 \\
\hline Total cholesterol $(\mathrm{mg} / \mathrm{dL})^{*}$ & $204.00(51.57)$ & $217.38(52.22)$ & $208.17(40.55)$ & 0.209 \\
\hline $\mathrm{HDL}-\mathrm{C}(\mathrm{mg} / \mathrm{dL})^{*}$ & $46.69(14.07)$ & $46.45(14.66)$ & $42.50(13.59)$ & 0.489 \\
\hline LDL-C $(\mathrm{mg} / \mathrm{dL})^{*}$ & $135.47(47.31)$ & $133.62(49.34)$ & $114.50(38.20)$ & 0.602 \\
\hline Triglyceride $(\mathrm{mg} / \mathrm{dL})^{\#}$ & $149(115,180)$ & $189(129,297)$ & $237(155,273)$ & 0.008 \\
\hline Statins use (\%) & 29.03 & 22.94 & 33.96 & \\
\hline
\end{tabular}

*mean (SD), ${ }^{*}$ median (IQR), ${ }^{\mathrm{a}} \mathrm{N}=1,137,{ }^{\mathrm{b}} \mathrm{N}=2,160,{ }^{\mathrm{c}} \mathrm{N}=392$

index, total cholesterol, HDL cholesterol and LDL cholesterol among these three groups of patients $(\mathrm{p}>0.05)$. However, systolic blood pressure, diastolic blood pressure and fasting blood glucose level were significantly different among these groups $(\mathrm{p}<0.05)$. The combined regimen users were likely to be prescribed with statins more frequently than ACEI-based regimen users and thiazide-based regimen users. (Table 1)

\subsection{Survival analysis}

There were $396(10.73 \%)$ patients who developed NOD over 6-years follow-up period. The median follow up time was 6 years. The total time at risk was 18,525.52 person-year. The incidence of NOD was 2.13 per 100 person-years.

In Kaplan Meier analysis, stratified by antihypertensive drugs regimens, the incidence rate of NOD at 6 years were $11.06(95 \% \mathrm{CI}, 9.19$ - 13.27), 14.17 (95\%CI, $12.48-16.05)$ and 14.17 (95\% CI, $11.53-17.35)$ for ACEI-based regimen, thiazide-based regimen and combined regimen respectively. (Table 2)

The crude Hazard ratio (HR) was 1.297 (95\%CI, $1.023-1.664)$ for thiazide-based regimen and $1.304(95 \% \mathrm{CI}, 0.973-1.748)$ for combined regimen, compared with ACEI-based regimen. In multivariate Cox regression analysis model for age, sex, body mass index, blood pressure, total cholesterol, HDL cholesterol, LDL cholesterol, triglyceride and statin use, the adjusted HR was $1.616(95 \%$ CI, $1.011-2.586)$ for thiazide-based regimen and $1.405(95 \% \mathrm{CI}, 0.806-2.449)$ for combined regimen compared with ACEI-based regimen. After adjusted with propensity score as covariate, the HR was 1.540 (95\% CI, 0.963 2.463) for thiazide-based regimen and 1.341 $(95 \%$ CI, $0.769-2.338)$ for combined regimen compared with ACEI-based regimen (Table 3)

\section{DISCUSSION}

In this study, we found that the incidence rate of NOD in ACEI-based regimen was lower than those in thiazide-based regimen, which is consistent with the previous studies indicating that NOD was lower in the ramiprilbased regimen than in the diuretic group ${ }^{13}$. Thiazide diuretics were associated with increased fasting blood glucose ${ }^{14-16}$, by affecting hypokalemia, which leads to decreasing in insulin secretion by $\beta$ cells in response to glucose $^{17}$ whereas ACEI may increase insulin sensitivity by increasing bradykinin level at the skeletal muscle, and promoting glucose transporter type 4 (GLUT4) protein expression in skeletal muscle and myocardium. This causes vasodilation of blood vessels and results in the increment of total perfused area and increased glucose uptake. ACEI can also reduce the promotion of inflammatory cytokines which reduce oxidativestress ${ }^{17,18}$.

Table 2. Incidence of new-onset diabetes.

\begin{tabular}{clll}
\hline & \multicolumn{3}{c}{ New-onset diabetes rate $(95 \% \mathrm{CI})$} \\
\cline { 2 - 4 } Survival time $(\mathrm{y})$ & ACEI-based regimen & Thiazide-based regimen & Combined based regimen \\
\hline 1 & $3.21(2.18-4.71)$ & $2.60(1.93-3.51)$ & $4.36(2.90-6.52)$ \\
2 & $4.98(3.69-6.70)$ & $4.62(3.70-5.76)$ & $5.47(3.84-7.76)$ \\
3 & $5.92(4.51-7.74)$ & $6.94(5.80-8.29)$ & $7.84(5.89-10.38)$ \\
4 & $7.71(6.12-9.68)$ & $8.49(7.22-9.97)$ & $9.48(7.35-12.19)$ \\
5 & $9.37(7.64-11.47)$ & $10.93(9.48-12.58)$ & $11.77(9.38-14.70)$ \\
6 & $11.06(9.19-13.27)$ & $14.17(12.48-16.05)$ & $14.17(11.53-17.35)$ \\
\hline
\end{tabular}


Table 3. Risk for incident NOD by antihypertensive drugs regimen.

\begin{tabular}{llclc}
\hline & \multicolumn{2}{c}{ Multivariate Adjustment } & \multicolumn{2}{c}{ Propensity Score } \\
\cline { 2 - 5 } & Adjusted HR ${ }^{*}(95 \% \mathrm{CI})$ & $p$-value & Adjusted HR ${ }^{\#}(95 \% \mathrm{CI})$ & $p$-value \\
\hline ACEI-based regimen & 1.00 & - & 1.00 & - \\
Thiazide-based regimen & $1.616(1.011-2.586)$ & 0.045 & $1.540(0.963-2.463)$ & 0.071 \\
Combined based regimen & $1.405(0.806-2.449)$ & 0.231 & $1.341(0.769-2.338)$ & 0.301 \\
\hline
\end{tabular}

*Adjusted by age, sex, body mass index, blood pressure, total cholesterol, triglyceride, HDL cholesterol, LDL cholesterol and statin use.

\#Adjusted by age, sex, body mass index, blood pressure, total cholesterol, triglyceride, HDL cholesterol, LDL cholesterol, statin use and propensity score

Due to lack of data and individual patient privacy, some confounding factors could not be controlled in data analysis. These include family history of diabetes, smoking status, alcohol consumption, gestational diabetes history, physical activity and poly ovarian syndrome which were risk factors of diabetes ${ }^{5-8,}, 19,20$. However, these confounding factors might not interfere the result because they are not associated with treatment preference.

Our study had several strengths. Firstly, it was the first one to investigate the association of antihypertensive drugs and NOD in a large Thai Isan patients with hypertension in clinical settings. Secondly, we conducted the new user design to avoid immortal time bias and assess time varying hazards and drug effects associated with treatment duration $^{21,22}$. Thirdly, we generated the propensity score to reduce the treatment selection bias due to non-random selection from routine clinical practice $^{23,24}$. Fourthly, we controlled for sex, age, body mass index, blood pressure, triglyceride, total cholesterol, HDL cholesterol, LDL cholesterol, and statin use while other Asian studies had different adjustment approaches ${ }^{9-11}$. For example, a retrospective longitudinal cohort study by Huang controlled many confounders, which is similar to this study, including age, sex, current smoking, family history of diabetes, body mass index, triglyceride, total cholesterol, HDL cholesterol, LDL cholesterol, and fasting blood sugar $^{9}$ while Jong GP controlled only for sex and age $^{10}$. Fasting blood glucose level was not included in the model as a predictor because FBS was used for NOD diagnosis. In addition, we used active comparator in our analysis while other studies used nonusers as comparing group ${ }^{9-11}$.

\section{CONCLUSIONS}

For the first study to investigate the association of antihypertensive drugs and NOD in Thai, our finding indicated that thiazidebased regimen and combined regimen were not associated with incident NOD compared with
ACEI-based regimen in hypertensive Thai Isan patients.

\section{ACKNOWLEDGEMENTS}

This work was supported by the faculty of pharmaceutical science, Ubon ratchathani university.

\section{Conflict of interest (If any)}

The authors declare no conflict of interests with this manuscript.

\section{Funding}

The Faculty of Pharmaceutical Sciences, Ubon Ratchathani University

\section{Ethical approval}

This study was approved by the ethics review committee for research involving human research subjects, Ubon ratchathani university (UBUREC-33/2559).

\section{Article info:}

Received January 14, 2019

Received in revised form September 18, 2019

Accepted December 2, 2019

\section{REFERENCES}

1. International Diabetes Federation. Key messages [ document on the internet]. Brussels: International Diabetes Federation; 2018 [cited 2018 Oct 1]. Available from: http://www.diabetesatlas.org/key-messages.html.

2. Shan AD, Langenberg C, Rapsomaniki E, Denaxas S, Pujades-Rodriguez M, Gale CP, et al. Type 2 diabetes and incidence of cardiovascular diseases: a cohort study in 1.9 million people. Lancet Diabetes Endocrinol. 2015;3(2):105-13.

3. Wee RY. Leading causes of death in Thailand [document on the internet]. Quebec: Worldatlas.com; 2017 [ cited 2018 Oct 1]. Available from: https://www. worldatlas.com/articles/leading-causes-of-death-inthailand.html.

4. Centers for disease control and prevention. Global health Thailand [document on the internet]. Alanta: Centers for disease control and prevention; 2018 [ cited 2018 Oct 1]. Available from: https://www.cdc.gov/ globalhealth/countries/thailand/default.htm.

5. The Inter Act Consortium. The link between family history and risk of type 2 diabetes is not explained by 
anthropometric, lifestyle or genetic risk factors: the EPIC-Inter Act study. Diabetologia. 2013;56:60-9.

6. Ratner RE. Prevention of type 2 diabetes in women with previous gestational diabetes. Diabetes Care. 2007;30(Suppl 2):S242-5.

7. Sullivan PW, Maorrato EH, Ghuschyan V, Wyatt HR, Hill JO. Obesity, inactivity and the prevalence of diabetes and diabetes - related cardiovascular cormobidities in the U.S., 2000 - 2002. Diabetes Care. 2005;28:1599-603.

8. Gambineri A, Patton L, Altieri P, Pagotto U, Pizzi C, Manzoli L, et al. Polycystic ovary syndrome is a risk factor for type 2 diabetes results from a long term prospective study. Diabetes. 2012;61:2369-74.

9. Huang CY, Ma T, Tien L, Hsieh YW, Lee SY, Chen $\mathrm{HY}$, et al. A retrospective longitudinal cohort study of antihypertensive drug use and new-onset diabetes in Taiwanese patients. Biomed Res Int. 2013;2013: 287696.

10. Jong GP, Chang MH, Tien L, Li SY, Liou YS, Lung CH, et al. Antihypertensive drugs and new-onset diabetes: a retrospective longitudinal cohort study. Cardiovasc Ther. 2009; 27(3):159-63.

11. Liou YS, Ma T, Tien L, Chien C, Chou P, Jong GP. Long term effects of antihypertensive drugs on the risk of new-onset diabetes in elderly Taiwanese hypertensives. Int Heart J. 2008;49(2):205-11.

12. Elliott WJ, Meyer PM. Incident diabetes in clinical trials of antihypertensive drugs: a network meta-analysis. The Lancet. 2007;369(9557):201-7.

13. Zidek W, Schrader J, Lüders S, Matthaei S, Hasslacher C, Hoyer J, et al. Ramipril-based versus diuretic-based antihypertensive primary treatment in patients with prediabetes (ADaPT) study. Cardiovasc Diabetol. 2012;11:1.

14. Brown MJ, Williams B, Morant SV, Webb DJ, Caulfield MJ, Cruickshank JK, et al. Effect of amiloride, or amiloride plus hydrochlorothiazide, versus hydrochlorothiazide on glucose tolerance and blood pressure (PATHWAY-3): a parallel - group, double - blind randomized phase 4 trial. Lancet diabetes endocrinol. 2016;4(2):90-2.

15. Stears AJ, Woods SH, Watts MM, Burton TJ, Graggaber J, Mir FA, et al. A double-blind, placebo-controlled, crossover trial comparing the effects of amiloride and hydrochlorothiazide on glucose tolerance in patients with essential hypertension. Hypertension. 2012;59:934-42.

16. Karnes JH, Gong Y, Arwood MJ, Gums JG, Hall KL, Limacher MC, et al. Alteration in fasting glucose after prolonged treatment with a thiazide diuretic. Diabetes Res Clin Pract. 2014;104:3.

17. Rizos CV, Elisaf MS. Antihypertensive drugs and glucose metabolism. World J Cardiol. 2014;6(7):51730.

18. Stas S, Appesh L, Sowers J. Metabolic safety of antihypertensive drugs: myth versus reality. Curr Hypertens Rep. 2006;8:403-08.

19. Wannamethee SG, Shaper AG, Perry IJ. British Regional Heart Study. Smoking as a modifiable risk factor for type 2 diabetes in middle-aged men. Diabetes Care. 2001;24:1590-5.

20. Baliunas OD, Taylor BJ, Irving H, Roerecke M, Patra J, Mohapatra S, et al. Alcohol as a risk factor for type 2 diabetes. Diabetes Care. 2009;32:2123-32.

21. Lund JL, Richardson DB, Sturmer T. The active comparator, new user study design in pharmacoepidemiology: historical foundations and contemporary application. Curr Epidemiol Rep. 2015;2(4):221-8.

22. Yoshida K, Solomon DH, Kim SC. Active-comparator design and new-user design in observational studies. Nat Rev Rheumatol. 2015;11:437-41.

23. Austin PC. An introduction to propensity score method for reducing the effect of confounding in observational studies. Multivariate Behav Res. 2011;46:399-424.

24. Johnson SR, Tomlinson GA, Hawker GA, Granton JT, Feldman BM. Propensity score methods for bias reduction in observational studies of treatment effect. Rheum Dis Clin N Am. 2018;44:203-13. 